\title{
Towards an ICT-based psychology: E-psychology
}

\author{
Athanasios Drigas *, Lefteris Koukianakis, Yannis Papagerasimou \\ NCSR 'Demokritos', Institute of Informatics and Telecommunications, Net Media Lab, Agia Paraskevi, 153 10, Athens, Greece
}

\section{A R T I C L E I N F O}

Article history:

Available online 18 February 2011

\section{Keywords:}

E-psychology

E-learning

ICTs

Cognitive science

E-diagnosis

Evaluation tests

\begin{abstract}
A B S T R A C T
Cognitive science is the scientific domain which studies, analyzes, simulates and infers for various aspects, functions and procedures of human mentality such as, thinking, logic, language, knowledge, memory, learning, perception and the ability to solve problems. E-psychology is in close relation with the cognitive science domain, but expands beyond it, as e-psychology is the efficient convergence of psychology and Information and Communication Technologies (ICTs). E-psychology offers a number of services such as supporting, diagnosis, assessment, therapy, counseling, intervention and tests through an effective exploitation of ICTs. This article presents a user-friendly, flexible and adaptive electronic platform, which supports both synchronous and asynchronous e-psychology activities through the use of informative and communicative tools and services, which can be adapted to support various methods of e-psychology activities. It is important to underline that e-psychology is not an alternative psychology field, but a resource to enhance the conventional psychology process.
\end{abstract}

(c) 2010 Published by Elsevier Ltd.

\section{Introduction}

The rapid advance of the Information and Communication Technologies (ICTs) and the Internet over the course of the last 15 years has affected a significant number of aspects of contemporary life including education. Nowadays, educational e-content can be found anywhere, anytime and to anyone who can connect to the Internet. Hence, it was only natural for universities and academic institutions to use this development to their advantage by providing on-demand web based education and training through course delivery platforms such as the Ariadne Web based learning environment and electronic textbooks through the use of authoring tools such as InterBook (Brusilovski, Eklund, \& Schwarz, 1998; Durm, Duval, Verhoeven, Cardinaels, \& Olivie, 2001).

A large number of sciences including Psychology have exploited the numerous capabilities and services of the Internet and have taken advantage of them to their benefit. More particularly, psychology uses the Internet in order to create programs for psychological intervention, assessment, orientation, and specialized counseling, as a means of prevention. The Internet not only constitutes a new communicative medium between the patient and the therapist but is also the future of psychology (González et al., 2000; Kenwright, Marks, Gega, \& Mataix-Cols, 2004; Lytras, Damiani, \& Ordóñez de Pablos, 2008; Lytras \& Ordóñez de Pablos, 2009; Marks, Shaw, \& Parkin, 1998; Richter \& Naumann, 2000; Riva, Molinari, \&

\footnotetext{
* Corresponding author. Tel.: +30 210 6503124; fax: +30 2106532910 .

E-mail addresses: dr@iit.demokritos.gr, dr@imm.demokritos.gr (A. Drigas), kouk@iit.demokritos.gr (L. Koukianakis), ypapa@iit.demokritos.gr (Y. Papagerasimou).
}

Vincelli, 2002; Vincelli, 1999; Vincelli \& Riva, 2002). An Internet environment designed and built for psychology purposes may use such tools as e-mail, chat rooms, discussion forums and audio and video conference for the communication and interaction of the therapist with the patient, tools which are also used for educational purposes in traditional e-learning environments (Albano, Gaeta, \& Salerno, 2006; Lytras, 2007; Lytras \& Garcia, 2008; Lytras \& Ordóñez de Pablos, 2007; Lytras \& Sicilia, 2005; Ordóñez de Pablos, 2002; Rodriguez Pérez \& Ordóñez de Pablos, 2003).

It is common knowledge that nowadays, the Internet is packed with information of uncertain quality and prestige. Taking this fact into account, it becomes more than apparent that it is absolutely essential to know how and where to extract useful and qualitative information from, regarding the object of ones interest. This becomes even more important and more vital when the object of interest regards health issues (Eysenbach, Powell, \& Kuss, 2002; Fogel, Albert, \& Schnabel, 2001; Gomella, 2000; Matthews, Camacho, Mills, \& Dimsdale, 2003). According to a recent study, real patients and supported individuals were found to trust entirely and resort to prominent and well-known websites in order to gather information regarding their health issue, without prior guidance by neither their therapists nor even by Internet experts. That is, they visited websites of major hospitals, health organizations as well as of government organizations in order to acquire information (García, Ahumada, Hinkelman, Muñoz, \& Quezada, 2004). Quality information with substantial validity and weight can have a positive psychological effect on patients. Hence, it becomes crucial that psychologists embed this new and innovative means of psychology in their practice and view it as a means to enhance the entire psychology process (Carlson \& Buskist, 1997; Fogel, 2004). 
Based on the aforementioned framework, an e-psychology platform was developed which was also based on the following principles. Firstly, it was decided that since the e-psychology platform is partially but principally addressed to supported individuals, which is a very sensitive social group, it was essential that the design was as user-friendly and user-centered as possible and according to the user needs.

Secondly, it was decided that the electronic content (e-content) and the tools of the platform should be modular and flexible. With this modularity and flexibility of both the e-content and the tools, the administrator has the capability to provide environments and services of different types. This is done, in order for the platform to support different target groups, various categories of supported individuals (depending on the type of support that they are receiving) and different categories of scientists (psychologists training, staff training) on the one hand and on the other, to support different psychological procedures. For instance, reusability can support a procedure that is based on behavioral psychological principles or it can organize an environment that is based on and embeds the diagnostic principles and tools of cognitive psychology.

Finally, the instructional methods that are embedded within the developed web-based e-psychology platform use most of the available modern multimedia and communicative technologies of the Internet and offer various modes for the delivery and presentation of the electronic content to the user.

\section{Abstract level description}

\subsection{E-psychology and e-learning: two interrelated concepts}

Psychology is distinguished into six major branches with respect to the cause of ones unusual behavior. All psychology branches refer to specific target groups based on unusual behavior problems. The method that each branch uses differs from the others while the processes (evaluation tests, diagnosis and therapy) are the same in all branches (Fig. 1).

Psychology uses learning and educational techniques in all the branches that it comprises (behavioral, psychodynamic, cognitive, etc.). These learning and educational procedures are used in various levels of the production and supporting of the psychological procedure. Thus, learning and education are used for the supported person level at the intervention procedure as well as at the level of organizing the psychology cycle and finally, at the level of the constant upgrade of the psychologists' and counselors' skills.

On the other hand, the e-learning procedure has proven that it can successfully use the ICTs for the supporting of learning, education and training in all the sectors and levels of the educational system as well as for lifelong learning and training.

The aforementioned e-learning techniques are embedded in the e-psychology system and it is proven that they can be used in the same successful way for the improvement of the psychology services provision cycle (to the end-user). The e-learning techniques used in the psychology services provision cycle are used in the following two axes.

Firstly, to support learning and education, which are indispensable in all the psychology branches and secondly, to entirely change the form of the provision of the psychological cycle services to the end-user, since the tools that are used for e-learning (in a new form and role now) are used to support the operations, procedures and services of the psychological cycle.

The possibility of supporting personal teaching - learning as well as supporting classes or larger entities like levels of learning or virtual schools finds also similarities and proportions to the psychological circle, with the possibility of supporting psychological consultancy in a personal way or in a small group, or in larger handling entities, such as therapy directions, or virtual psychology health centers.

\subsection{An e-psychology environment}

A complete e-psychology environment can offer and support a large number of the operations and services of the psychological cycle such as tests, evaluation, diagnosis, support and intervention between the supported individuals and the psychologists while simultaneously, it offers all the necessary learning and educational procedures and services to this heterogeneous community of recipients (psychologists, counselors and supported individuals).

Such an e-psychology system usually aims to supplement and improve parts of the traditional psychology services provision cycle without substituting the personal contact between the psychologist and the supported individual.

The aim of the discussed application is the integration and convergence of the ICTs with the traditional psychology services for

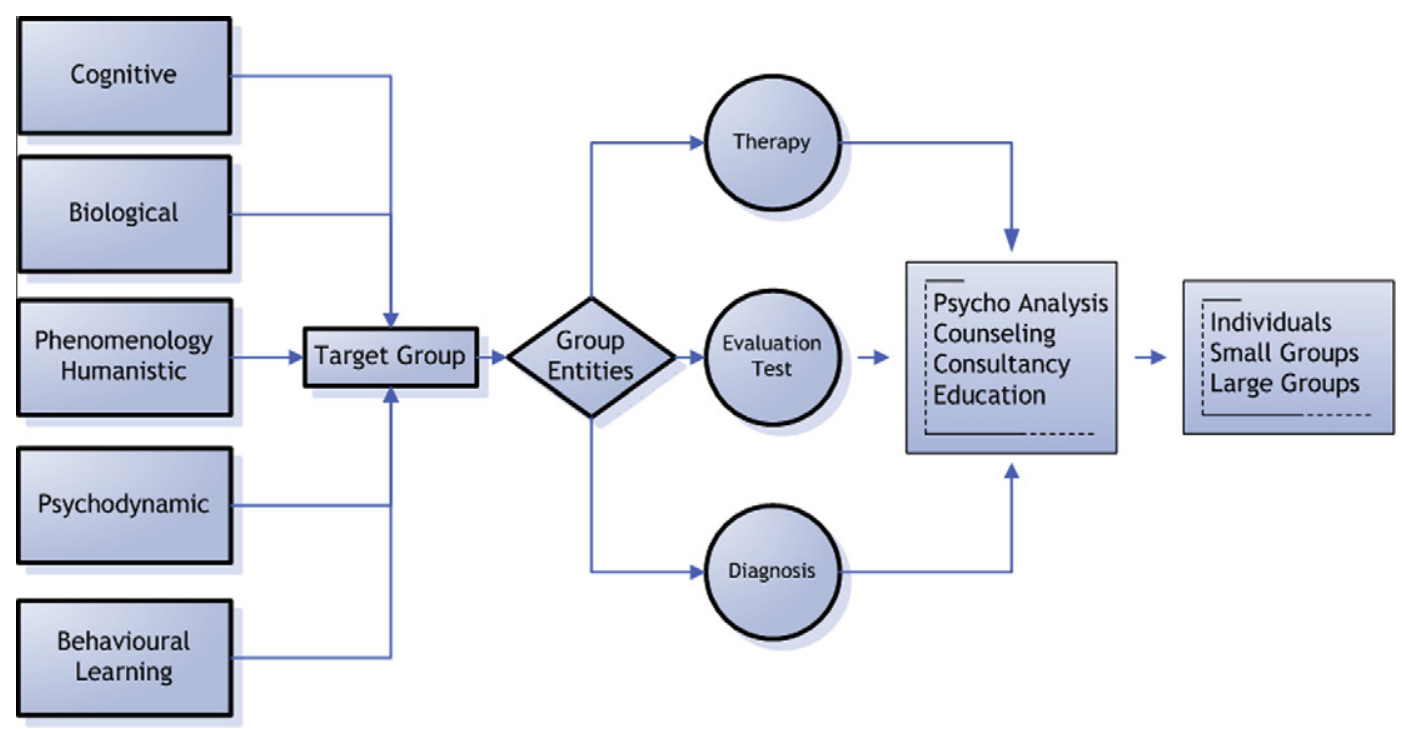

Fig. 1. Psychology services here. 


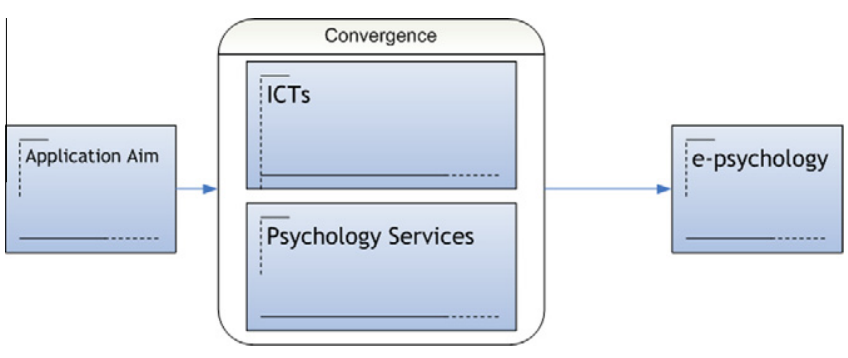

Fig. 2. E-psychology abstract logical structure.

the development of a system that will support e-psychology activities (Fig. 2).

The result of the ICTs integration with the traditional psychology services is depicted more analytically in the following Fig. 3. This integration of the psychology branches and services in a web-based environment is available to the Internet user. The methods and processes that each branch follows are materialized through electronic tools such as: discussion forums, message boxes, chat, video and audio conference, which will be analyzed in detail in the next sections.

\section{E-psychology: an overview}

\subsection{Modeling psychology procedures}

The first step that is required in order to develop and design an e-psychology environment is to model the operations and procedures of the psychology cycle (Fig. 4). The most important step in the psychological services provision procedure is diagnosis. Diagnosis, as it is presented below is based on the one hand, on the diagnostic criteria, which are associated with DSM-IV and ICD-10, while on the other hand it utilizes and is based on diagnostic instruments such as interview, psychometric tests and observation. The result of all of the above is the production of the assessment report.

It is obvious that these distinguished steps of the diagnosis procedure are the main structural ingredients with which it is possible to create an embedded system in an e-psychology environment, which performs the operations of diagnosis support (e-diagnosis).

Following the modeling of diagnosis, it is very important to underline the importance of modeling the stages and the procedures of therapy - treatment to the process of designing and realizing an e-psychology environment.

Based on the assessment report that was derived through the diagnosis process, the therapy - treatment process follows the stages that are depicted above in Fig. 4. The basis for the development of these stages for this process is the treatment plan report, which consists of the treatment approaches and the treatment modality. With the term treatment approaches we refer to the various therapeutic and theoretical approaches of psychology such as cognitive, behavioral, psychodynamic and existential. On the other hand, with the term treatment modality we refer to the various means of intervention such as one to one, small group, family and couples.

Finally, at the last stage of the modeled therapeutic process, the treatment report is produced as well as the discharge report and finally the patient outcome.

\subsection{E-psychology process cycle}

Following the discussion about modeling the operations and procedures of the psychology cycle, this chapter presents the epsychology process cycle (Fig. 5), which is realized in our platform

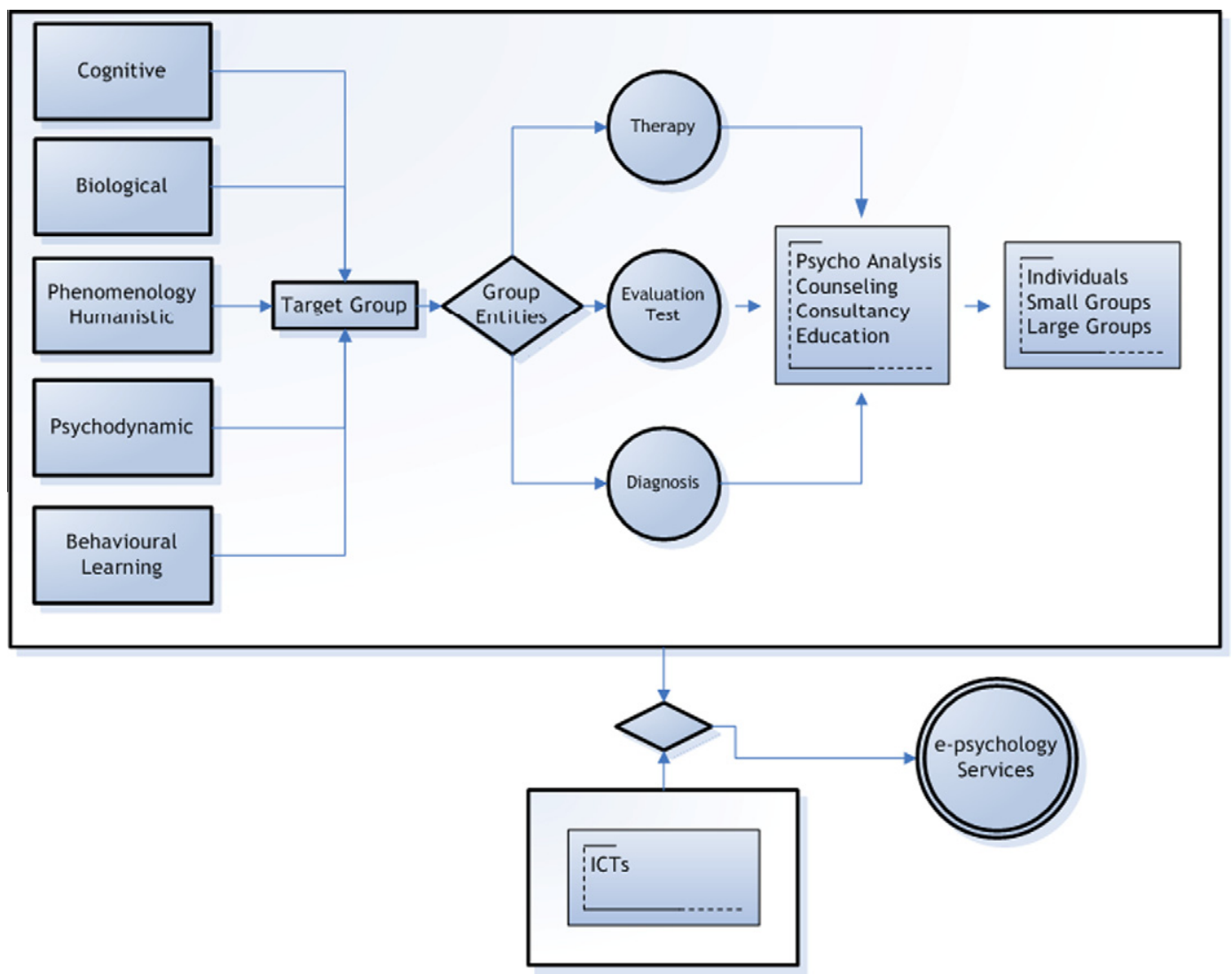

Fig. 3. E-psychology logical structure. 


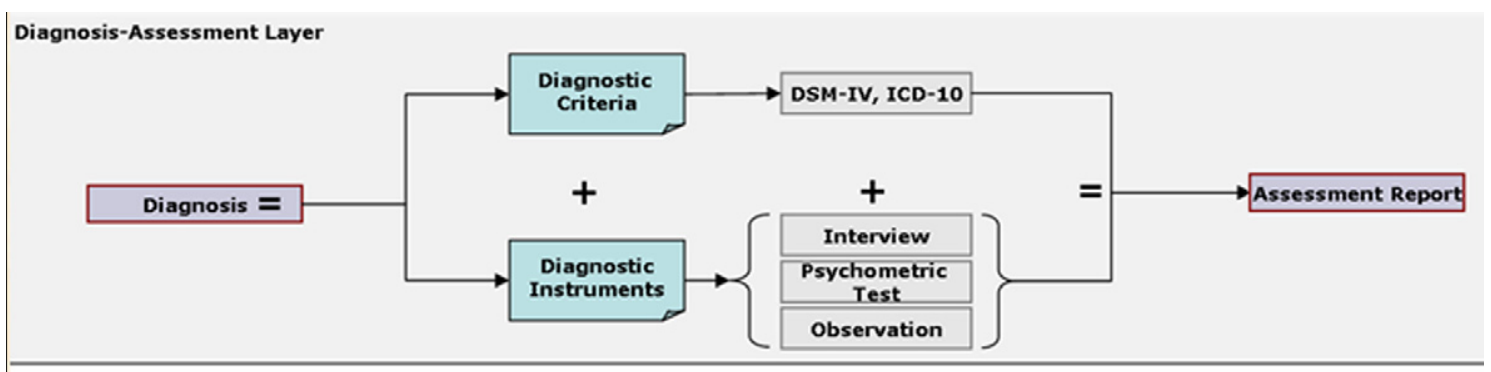

Psychology $=$ Diagnosis + Therapy $=$ Describe, Explain, Predict, Control, Improve

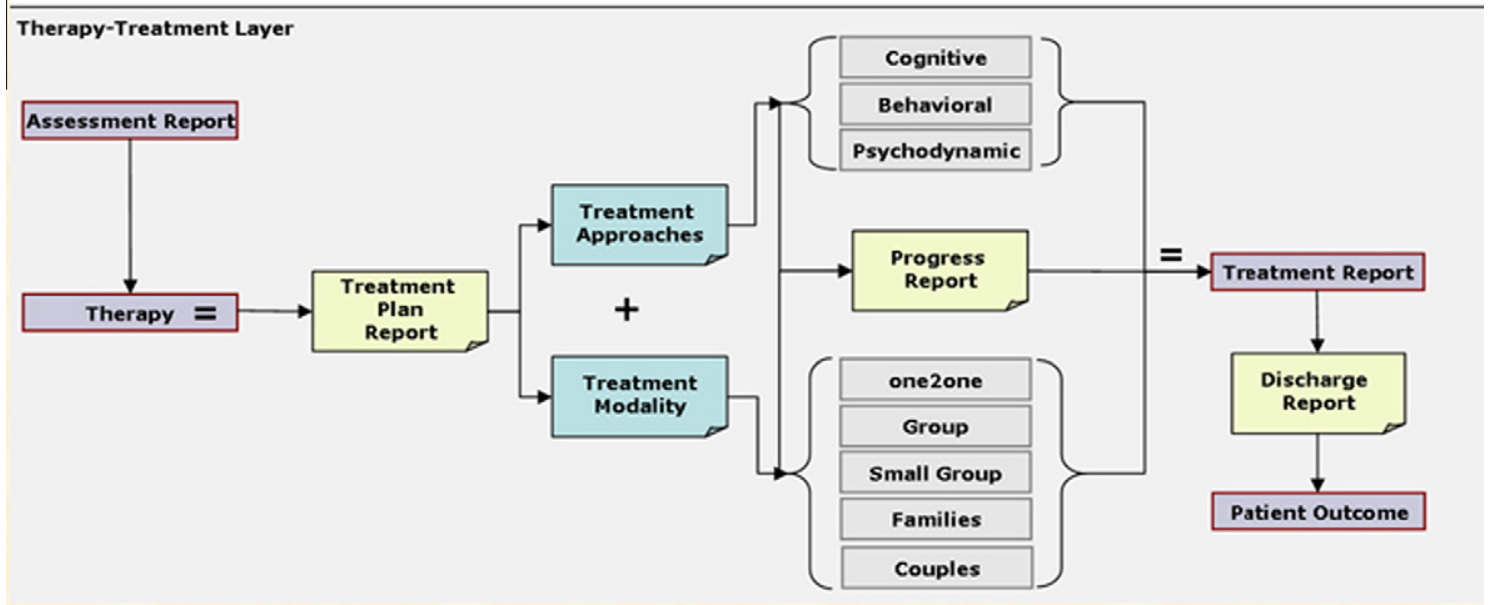

Fig. 4. Psychology model diagram.

and which was the basis for the development of the e-psychology environment (presented in Section 4).

The supported person (SP) has access to the registration department through a registration form over a secure socket layer (SSL) and his/her application becomes accepted after the necessary validation process. Following this, a temporary supported person page is created and through certain electronic procedures such as psychometric tests and interviews the assessment report is produced.

Furthermore, through the creation of the supported person's history folder and the appropriate review, the supported person's personal information is inserted into the appropriate virtual clinic department where suitable treatment modalities are chosen and applied through suitably designed treatment plans. At this stage, the informative tools that support and realize the aforementioned procedures are the Supported Person calendar, his/her health files, his/her personal information, the psychologist, the health library and a forum.

At the end of the e-psychology process cycle the patient e-outcome is derived provided that all the necessary conditions of the treatment plan report, progress report and discharge report are met successfully.

\subsection{E-psychology and e-learning tools and services}

The presented e-psychology process cycle is realized through special information and communication technologies tools and services. The design of these tools and services was based on existing web services, such as discussion forums, chat, message boxes and e-libraries, which are widespread in the public web community. These tools and services are divided into two major categories: the informative and the communicative tools and services. The former are divided further into two subcategories: the informative and the supportive tools and services, which include tools and services related to the supporting material and its presentation within the e-psychology environment. Similarly, the latter (the communicative tools and services), are also divided into two subcategories: the synchronous (real-time) and the asynchronous (non real-time) tools and services, which include tools and services that allow the communication between different user groups (users belonging to a different session level) (Fig. 6).

The aforementioned ICT tools and services were used for the development of the e-psychology platform imprinting the traditional learning and psychology processes with synchronous and asynchronous learning and psychology tools and services in the e-psychology platform.

The informative tools and services offer electronic tests (e-tests) and auditing tools, automated diagnosis, electronic content and knowledge for instant access, online databases hosting frequently and non-frequently found cases, tools to trace the impact and the progress of any treatment or supporting method, etc. On the other hand, the communicative tools and services offer alternative paths of communication (real-time and non real-time) between the psychologist and the supported person, in order to trace the impact and the progress of therapy, supporting, consulting and intervention.

The e-psychology platform offers the possibility of management of these tools and services according to the user groups' permission. More explicitly, the informative tools and services comprise the following: web directory, supported material, schedule, glossaries, references, video and audio lectures, events calendar, news, announcements and mailing lists. On the other hand, the supportive tools and services consist of: exercises, diagnosis and diagnosis tests. As far as the communicative tools and services are concerned, in the synchronous subcategory these are: video and audio conference, instant messaging, chat and telephony. One the other hand, the asynchronous tools and services include: e-library, discussion forums, message boxes, e-mail and finally, video and audio e-mail (Fig. 7). 
Supported Person

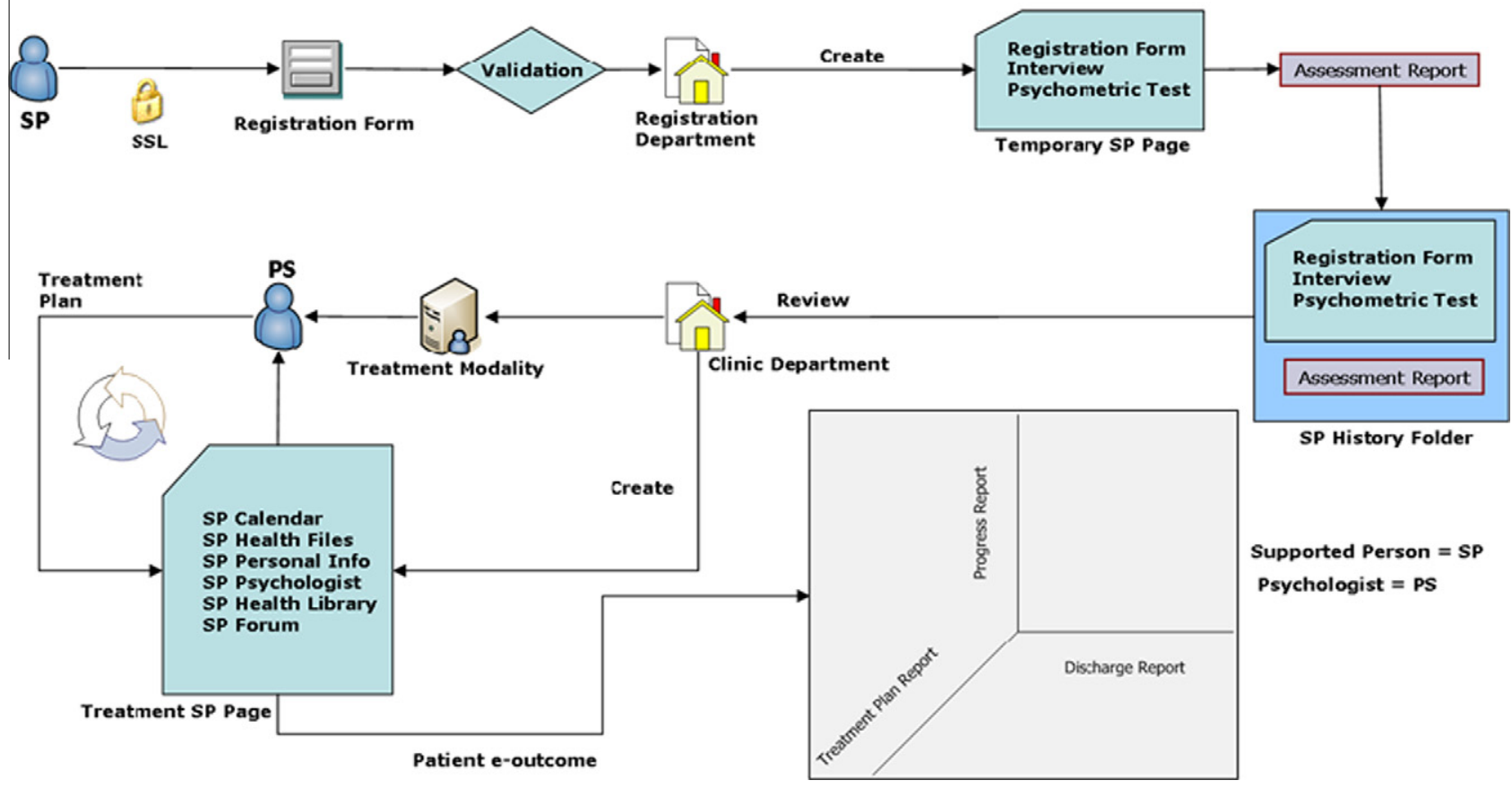

Fig. 5. E-psychology process cycle diagram.

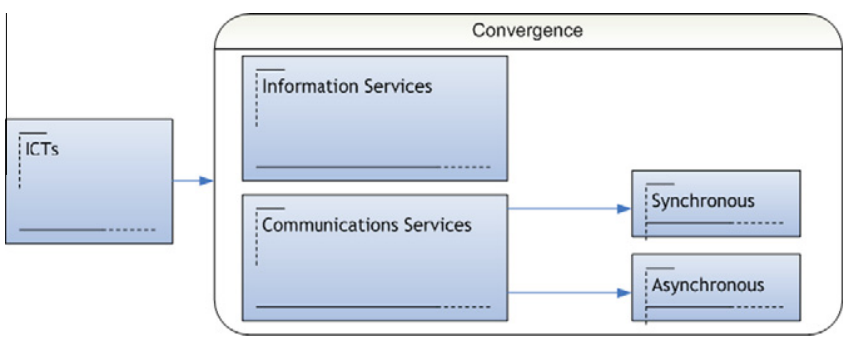

Fig. 6. Information and communication technologies (ICT) tools and services.

In addition, the user levels and user interfaces of the e-psychology platform comprise the following four levels: the administrator, the therapist - psychologist, the patient - supported person and finally the visitor who support similar actions and have similar roles with the e-learning user levels namely, administrator, instructor, student and user. Finally, the seminars, classes, courses and the educational material from the e-learning circle are supported by the same tools and in similar procedures with the therapeutic entities, small groups, therapeutic processes, and supporting material, from the e-psychology circle.

The specifications of the user levels, the e-content and the etools in an e-psychology platform can be easily implemented through a simple correspondence of the psychology ontologies to the generic e-learning ontologies.

E-psychology and e-learning have a very close interrelation. More particularly, the way that e-learning is related to e-psychology, is depicted below (Fig. 8). It is obvious that the roles of the "instructor" and the "student" are transformed into the roles of the "psychologist" and the "supported individual" respectively. The "classes" are transformed into "small therapy groups" and the "courses" into "supporting material". The "consulting material" (examples, exercises, multiple choice tests) correspond respectively to diagnosis, educational exercise for treatment and diagnostic tests. Finally, as it has been previously mentioned all the tools and services such as web directory, glossary, references, video and audio lectures, events calendar, news, announcements, mailing lists, e-library, message box, e-mail, video and audio conference, discussion forums, instant messaging, chat, and telephony can be easily applied to both platforms.

\section{E-psychology platform presentation}

\subsection{E-Psychology Platform Structure}

Based on the discussion in Section 3, we have developed an epsychology platform which is abstractly depicted in (Fig. 9). The environment supports the operations of e-diagnosis and e-therapy that lead to the successful coverage of the aims that were set according to the followed psychological approach, in order to finally reach the e-outcome (provided that the aims are met successfully).

These operations support and refer to the user target groups. These comprise the supported persons on the one hand and on the other the therapists - psychologists, who receive support through the e-psychology environment in order to execute their task in the best possible way. The supported persons comprise individuals, groups, small groups, families and couples while the individuals can be either adults, children or elderly people.

The module that refers to training plays a very important role in the structure of the e-psychology platform. Training is a very important tool in the e-psychology field as it is used not only in the various fields of psychology for the support of the supported persons, but also for the support and constant upgrade of the cognitive skills of the therapists - psychologists and the formation of the way that they treat both the supported persons as well as the various procedures within the e-psychology environment.

Beyond the therapists - psychologists and the supported persons, the training module of the e-psychology platform constitutes a very powerful tool for the training of researchers and students who are given permission to access the e-psychology platform. Fi- 


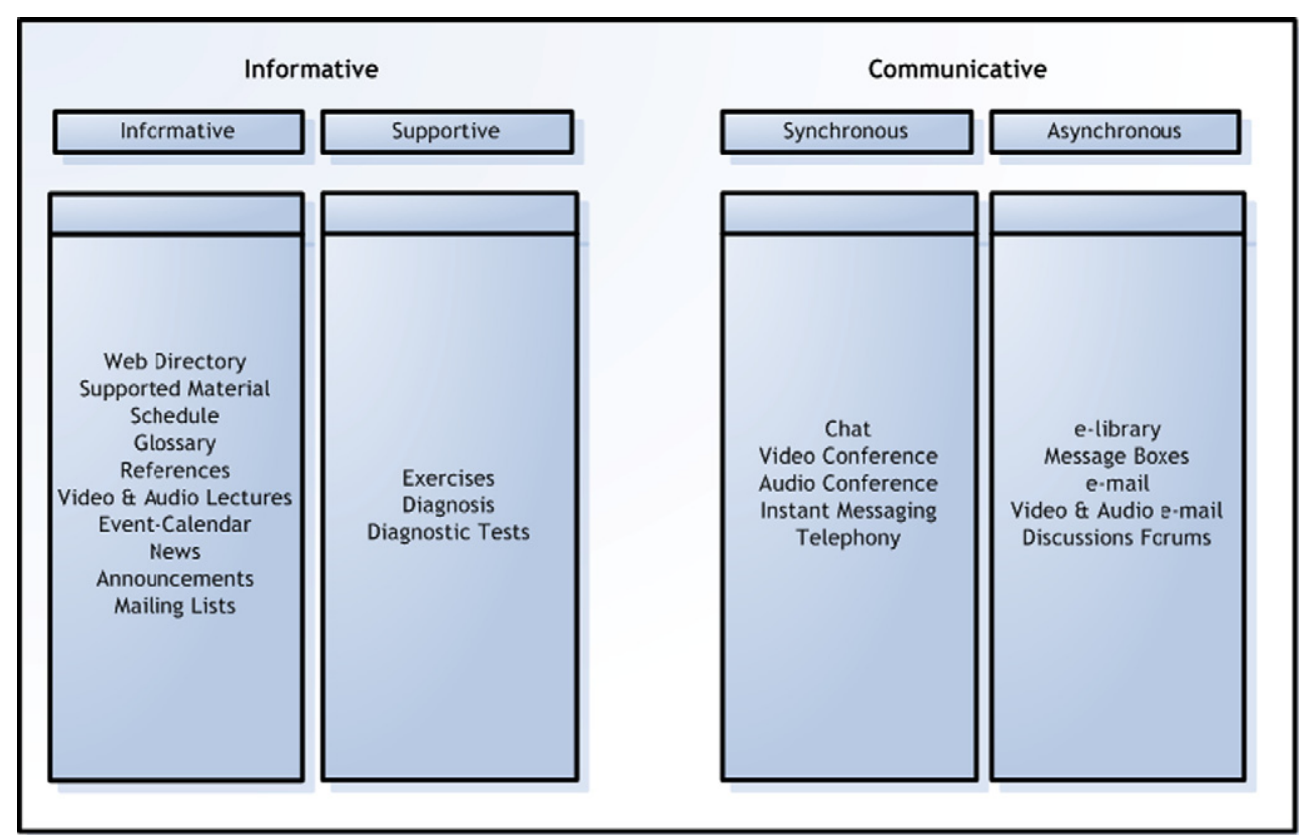

Fig. 7. Informative and communicative tools and services.

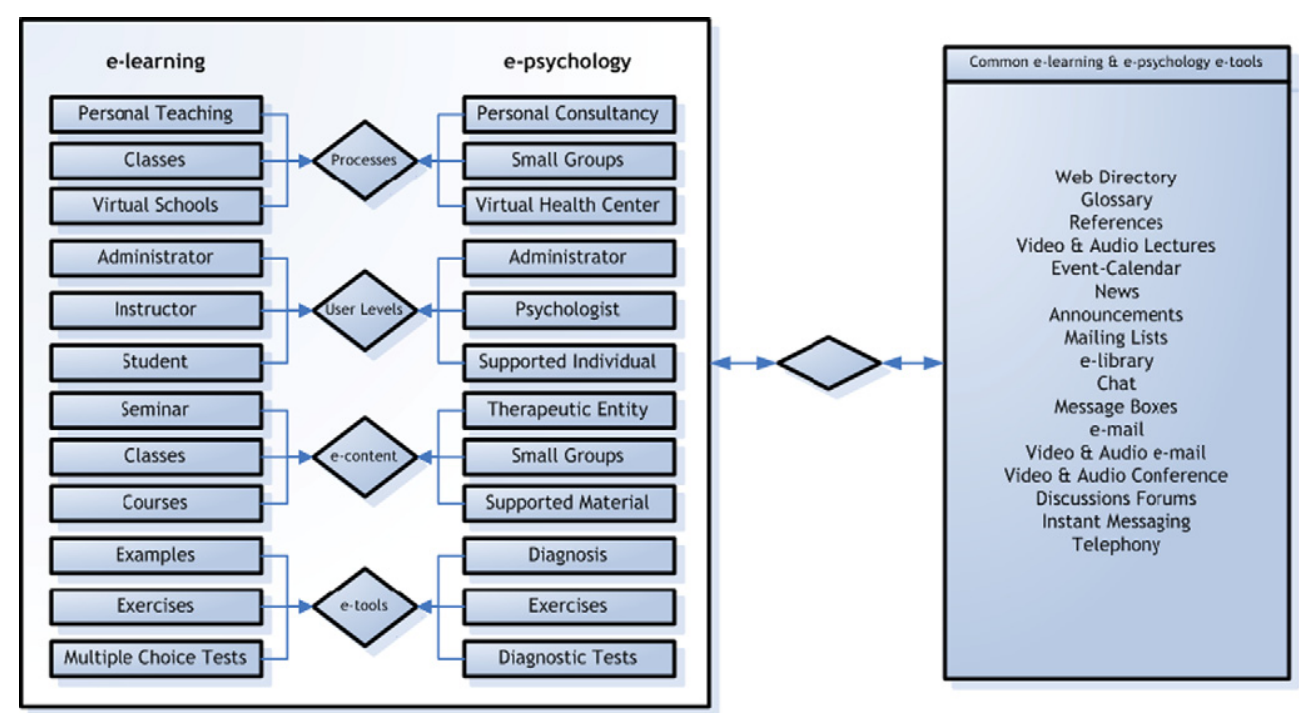

Fig. 8. E-learning and e-psychology interrelation.

nally, the importance of the training modules' role in the training of the common user (mean citizen) should not be overlooked as it informs and trains him/her on various interesting subjects regarding everyday psychological health such as stress control, possible perception or even behavioral deflections in children, memory issues with adults and the elderly and finally, substances abuse etc.

\subsection{E-psychology platform user levels}

The discussed e-psychology platform comprises four different user levels namely the administrator, the psychologist - therapist, the patient - supported individual and finally, the visitor. Each of these user levels has a different role and different permission levels to both the informative and the communicative tools and services of the e-psychology platform.

The role of the administrator is to manage the e-psychology platform using the administrative tools of the system. These tools give the administrator the capability to hand permission to the other users of the e-psychology platform to access its various tools and services, depending on their needs. Finally, the administrator is in constant communication with the therapists - psychologists regarding their sessions with their patients and their progress.

Moreover, the role of the therapist - psychologist is to create and organize the electronic content (e-content) of the e-psychology platform in terms of its delivery to the patients - supported individuals as well as to anyone who is interested in viewing it. In particular, general information regarding psychological issues is uploaded to the system but more importantly, the electronic content of the sessions that the therapist - psychologist engages in with a patient is also uploaded to the e-psychology system. The scope of this is to enable the patients - supported individuals to revise the session in their own time in order to have a better perception of it. Finally, there is also the online communication, which aims at the further interaction of the two parts and the reply of possible questions that may rise after the end of a session. 


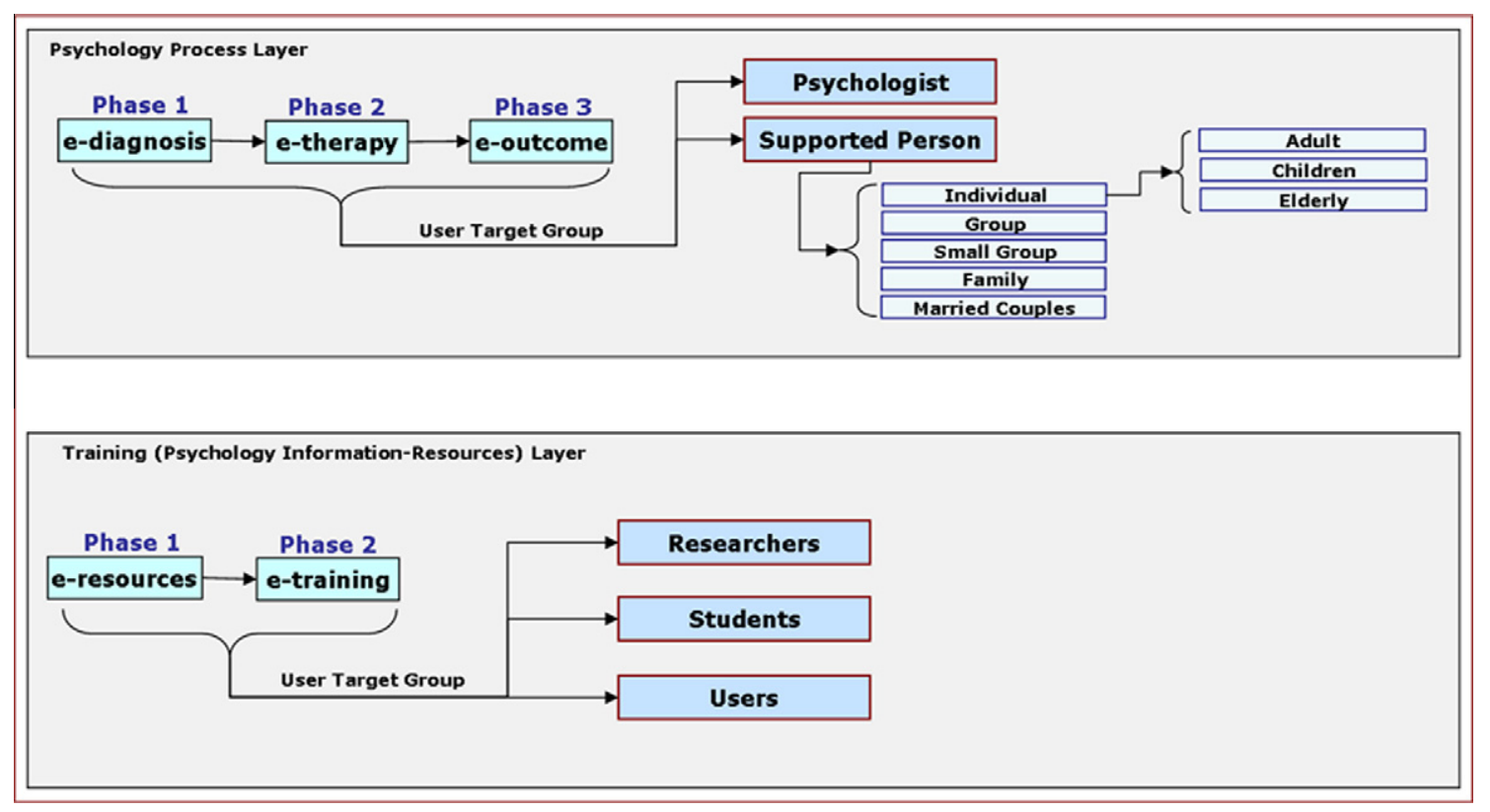

Fig. 9. Abstract presentation of the e-psychology platform structure.

Furthermore, the role of the patients - supported individuals is that of the regulator of the e-psychology platform. That is to say that it is the patients - supported individuals that view the e-content, engage in the online sessions with the therapists - psychologists and hence it is up to them to determine whether the epsychology platform is worth while or not. Additionally, the patients - supported individuals have full access to all the information and the services that are provided by this e-psychology platform.

Finally, the visitor (common citizen) has the unique opportunity to access the e-content of the discussed e-psychology platform. This means access to consulting material, general sessions and discussion forums where the visitor can go through discussions between the therapists - psychologists and the patients supported individuals for purely informative and educational purposes. This innovation is in accord with the principle information and knowledge for all' which conforms to the knowledge society strategies. It must be noted that these discussions between the therapists - psychologists and the patients - supported individuals are generic and non confidential. It is obvious that the simple visitors of the e-psychology platform do not (and can not) have the ability to access the e-content that has to do with confidential sessions and discussions between therapists - psychologists and patients - supported individuals. This personal information is protected and can only be accessed by the other three user levels (administrator, therapist-psychologist, patient-supported individual) who are authorized to do so.

\section{Future work - e-psychology and disabled individuals}

The presented e-psychology platform constitutes only the first stage of an integrated e-psychology environment. The main future goal regarding the discussed e-psychology platform is the development of an up-to-date integrated e-psychology environment, which will support e-psychology activities for the needs of individuals with visual and hearing impairments through the use of ICT tools and services.

E-psychology engages in activities such as teaching, learning, supporting, consulting, intervention and interview, aiming at enhancing the skills and smoothing the handicaps of disabled people. Through the efficient use of ICT tools and services, such e-psy- chology environments, analyze and understand in full the handicaps of this special group of people, achieving hence to cover both their special and communicative needs. This will ensure their equal access to information, knowledge, education and employment. Moreover, this future electronic environment will comply with the "Design for All" and "Universal Accessibility" principles, rendering it user-friendly, flexible and adaptive for all.

Within this integrated e-psychology platform, lessons in the Sign language will be developed and used, which will deal with the use and exploitation of ICTs for the hearing impaired. The development of these lessons will be based on multimedia assistive technologies such as visual material, streaming digital video and video conference for the hearing impaired, while for the visually impaired the development of the aforementioned lessons will be based on such assistive technology as Braille terminals, screen readers, screen magnifiers, speech synthesizers, and voice/audio input software will be embedded within the system to support the e-inclusion of this special group of people.

The implementation of this integrated platform will be based on two axes-ideas. The first deals with the use of ICTs as a tool in order to expand the communication abilities of the disabled and as an intervention tool. The second deals with the use of ICTs as learning objects in order to exploit them and enhance their vocational life, ensuring their equal access to continuous vocational training without any form of discrimination, developing their enterprising and competitiveness skills through ICTs, expanding their vocational horizon and finally, developing their professional skills through the ICT tools. Furthermore, the environment will support both synchronous and asynchronous activities of teaching-learning via communicative and informative tools and services, which can be adapted to support various methods of teaching-learning. Finally, the environment will support self-study learning as well as teacher moderated study via audio or video conference usage.

\section{Pilot implementation}

The discussed e-psychology platform was developed under the "Conditions Improvement of Inclusion to Educational System of Individual with Multiple Handicaps" framework of the Greek research program, which was funded by the O.P.E.I.P.T. European community program (Operational Program of Education and Initial 
Professional Training). The project's main objective was to train special education teachers on multiple handicaps issues. The work that was carried out during the pilot implementation period included two seminar periods: the first was the "training" seminar period while the second was the "specialization" seminar period. The former included the training of special education teachers in physical classrooms while the latter included training both in physical classrooms as well as with the use of e-learning. This electronic environment was parameterized and configured properly in order to conform to the initial project specifications and needs. In this way, it assured the distant training form and also it supported the dissemination of new ideas and knowledge in the special education community.

\section{Conclusions}

It is common knowledge that cognitive science is the scientific domain which consists of various categories of different research and knowledge fields, such as neuroscience, specific biology fields, specific informatics fields like artificial intelligence and neural networks and psychiatry fields. The common ground in these different sciences within the cognitive science domain is the study, research, analysis, simulation, inference, knowledge creation, etc., for various aspects, functions and procedures of human mentality, such as thought, knowledge, language, memory, learning, perception and the ability of solving problems. E-psychology is in a close relation with the cognitive science domain, but as it has been previously mentioned, it expands beyond it, as e-psychology is the convergence of psychology as a totality, with the informatics science, or in other words, it is the invasion of information and communication technologies (ICTs) within the psychology domain.

The era of information and knowledge society, became a reality or came into existence, because of the penetration of ICTs into the overall human (personal and social, scientific and non), activities. For several late years, we are referring, to various new fields like, e-learning, e-commerce, e-health, e-culture, e-government, e-testing, e-inclusion, e-democracy, e-politics, etc. Within the priorities and policies of EU, it is also known that we have a set o policies, which is known as e-Europe. E-Europe delimits goals, actions and procedures in order to improve the penetration and exploitation of ICTs and e-services, within every social and governmental, reality. ICTs are expected to improve, accelerate, and increase the quality, productivity, gain, and even satisfaction of the end-users, in every e-service. In this article we explored the various aspects of e-psychology.

The incorporation of information and communication technologies (ICTs) as well as of Internet technologies within the traditional psychology process cycle results in what is commonly known as epsychology, which was the main topic of this article. Although epsychology is an innovation and a step forward for traditional psychology, it was underlined from the start that under no circumstances does it substitute, replace or undermine the traditional psychology process but acts more as a means to enhance it and to complement it.

E-psychology in its broad sense enables the therapists - psychologists as well as the patients - supported individuals to use and incorporate technology in their sessions bringing the latter to a whole new level. While carrying on their traditional sessions both parts have the opportunity to simultaneously experiment with this new electronic process in order to deduce useful conclusions and to make the whole experience of a virtual session more appealing and beneficiary for both parts. E-psychology offers powerful infrastructures, tools and services in order to deliver their advanced quality to the end-users who are mainly the psychologists and the supported persons.
This e-psychology platform was based on modern informative and communicative tools and services as is the case in many modern e-learning platforms. More particularly, it was designed and developed very meticulously in an effort to provide an innovative platform that was user-centered, user-friendly, modular and flexible. In addition, the aim was to exploit in full all the capabilities of contemporary Internet technologies for the benefit of both therapists - psychologists and patients - supported individuals but mostly in an effort to primarily cover as much as possible the psychological needs (regardless of the field) of the aforementioned users of the e-psychology platform.

\section{References}

Albano, G., Gaeta, M., \& Salerno, S. (2006). E-learning: A model and process proposal. International Journal of Knowledge and Learning, 2(1/2), 73-88.

Brusilovski, P., Eklund, J., \& Schwarz, E. (1998). Web-based education for all: A tool for development adaptive courseware. Computer Networks and ISDN Systems, 30(1-7), 291-300.

Carlson, N., \& Buskist, W. (1997). Psychology: The science of behavior. Allyn and Bacon, Inc..

Durm, R., Duval, E., Verhoeven, B., Cardinaels, K., \& Olivie, H. (2001). Extending the ARIADNE web-based learning environment. In Proceedings of the 13th World Conference on Educational Multimedia, Hypermedia and Telecommunications (pp. 1932-1937). Tampere, Finland.

Eysenbach, G., Powell, J., \& Kuss, O. (2002). Empirical studies assessing the quality of health information for consumers on the World Wide Web: A systematic review. The Journal of the American Medical Association, 287(20), 2691-2700.

Fogel, J. (2004). Internet breast health information: Use and coping among women with breast cancer. CyberPsychology and Behavior, 7(1), 59-63.

Fogel, J., Albert, S., \& Schnabel, F. (2001). Health information on the internet. The Journal of the American Medical Association, 285(20), 2612-2621.

García, V., Ahumada, L., Hinkelman, J., Muñoz, R., \& Quezada, J. (2004). Psychology over the Internet: Online experiences. CyberPsychology and Behavior, 7(1), 29-33.

Gomella, L. (2000). The wild, wild web: Resources for counseling patients with prostate cancer in the information age. Seminars in Urologic Oncology, 18(3), $167-171$.

González, G. M., Winfrey, J., Sertic, M., Salcedo, J., Parker, C., \& Mendoza, S. (2000). A bilingual telephone-enabled speech recognition application for screening depression symptoms. Professional Psychology: Research and Practice, 31(4), 398-403.

Kenwright, M., Marks, I., Gega, L., \& Mataix-Cols, D. (2004). Computer-aided selfhelp for phobia/panic via internet at home: A pilot study. The British Journal of Psychiatry, 184, 448-449.

Lytras, M. D. (2007). Teaching in the knowledge society: An art of passion. International Journal of Teaching and Case Studies, 1(1/2), 1-9.

Lytras, M. D., Damiani, E., \& Ordóñez de Pablos, P. (2008). Web 2.0: The business model. Springer.

Lytras, M. D., \& Garcia, R. (2008). Semantic Web applications: A framework for industry and business exploitation - What is needed for the adoption of the Semantic Web from the market and industry. International Journal of Knowledge and Learning, 4(1), 93-108.

Lytras, M., \& Ordóñez de Pablos, P. (2007). Red Gate Corner: A Web 2.0 prototype for knowledge and learning concerning China business and culture. International Journal of Knowledge and Learning, 3(4 and 5), 542-548.

Lytras, M. D., \& Ordóñez de Pablos, P. (2009). Social Web Evolution. Integrating Semantic Applications and Web 2.0 Technologies. IGI-Global.

Lytras, M. D., \& Sicilia, M. A. (2005). The knowledge society: A manifesto for knowledge and learning. International Journal of Knowledge and Learning, 1(1/2), $1-11$.

Marks, I., Shaw, S., \& Parkin, R. (1998). Computer-aided treatments of mental health problems. Clinical Psychology: Science and Practice, 5(2), 151-170.

Matthews, S., Camacho, A., Mills, P., \& Dimsdale, J. (2003). The internet for medical information about cancer: Help or hindrance? Psychosomatics, 44(2), 100-103.

Ordóñez de Pablos, P. (2002). Knowledge management and organizational learning: Typologies of generic knowledge strategies in the Spanish manufacturing industry from 1995 to 1999. Journal of Knowledge Management, 6(1), 52-62.

Richter, T., \& Naumann, G. (2000). Computer-based assessment of reading skills. In Proceedings of Computers in Psychology Conference (CiP 2000). York, UK.

Riva, G., Molinari, E., \& Vincelli, F. (2002). Interaction and presence in the clinical relationship: Virtual reality (VR) as a communicative medium between patient and therapist. IEEE Transactions on Information Technology in Biomedicine, 6(3), 198-205.

Rodriguez Pérez, J. M., \& Ordóñez de Pablos, P. (2003). Knowledge management and organizational competitiveness: A framework for human capital analysis. Journal of Knowledge Management, 7(3), 82-91.

Vincelli, F. (1999). From imagination to virtual reality: The future of clinical psychology. CyberPsychology and Behavior, 2(3), 241-248.

Vincelli, F., \& Riva, G. (2002). Virtual reality: A new tool for panic disorder therapy. Expert Review of Neurotherapeutics, 2(3), 377-383. 